\title{
Optimed, le Réseau de santé genevois: bilan de trois années d'activité
}

\author{
J.-F. Burkhalter
}

\section{Historique}

Conscient de l'évolution des coûts de la santé et soucieux des conditions socio-économiques précaires que le canton de Genève a connues au début des années 1990 avec un record national du montant de la prime d'assurance-maladie et du taux de chômage, le Groupe des médecins internistes genevois a cherché, dès 1994, des moyens visant à contenir l'augmentation constante des coûts de la santé.

Selon les assureurs, les facteurs prépondérants de cette augmentation étaient liés, entre autres, au tourisme médical des patients, à leur recours abusif aux spécialistes et à une prescription excessive d'examens complémentaires par les médecins-traitant au détriment d'une écoute adéquate, aucune de ces affirmations n'étant étayée par des données chiffrées.

Les médecins ressentaient individuellement un malaise d'autant plus grand face à cette situation que leurs honoraires, fixés dans un tarifcadre par le gouvernement genevois, n'avaient subi aucune modification depuis 1991. Parmi les solutions envisagées, la plus prometteuse semblait être la mise sur pied d'un réseau de médecins de premier recours. La création d'un réseau s'inscrit dans les formes d'assurances prévues à l'article 41, alinéa 4 de la LAMal et aux articles 99 à 101 de l'OAMal.

\section{Partenaires}

Il faut rappeler, qu'aux débuts des années 1990, des assureurs ont créé des réseaux «en catimini» ou envisageaient de le faire en imposant leurs conditions aux médecins.

Le Groupe des médecins internistes a proposé aux généralistes et aux pédiatres de s'associer à ce projet. Ceux-ci ont répondu favorablement.

Les internistes, les généralistes et les pédiatres souhaitaient mettre sur pied un réseau dont ils contrôleraient le fonctionnement et les flux financiers. C'est dans cet esprit qu'ils ont rédigé et proposé, en novembre 1995, à tous les assureurs actifs à Genève une Convention réglant les relations entre médecins de premier recours et les caisses-maladie. Le Groupe Cosama, avec lequel des contacts informels avaient été établis et dont faisaient partie Assura, CMBB, la Caisse-maladie de la fonction publique, Fama, Futura, Hermes, La Caisse Vaudoise, Mutuelle Valaisanne, Philos, Supra, Universa et Intras ont immédiatement manifesté leur intérêt. Des négociations assez laborieuses ont été entamées en décembre 1995. Très rapidement, les pédiatres ont quitté la table des négociations, estimant que leur participation à un réseau n'entraînerait pas de diminution de leurs coûts. La Convention a été signée le 28 mai 1996. Afin de piloter ce Réseau, les internistes et les généralistes se sont regroupés en juin 1996 dans une structure appelée «Réseau des médecins internistes et généralistes genevois» sous le sigle RMIGG.

\section{But}

A la recherche d'une stabilisation des frais médicaux, ce Réseau propose une relation étroite entre les patients, les médecins et les caissesmaladie dans l'intention de maîtriser les coûts de la santé sans diminuer la qualité des soins, par un contrôle plus rigoureux des prestations médicales, de la prescription d'examens complémentaires et des dépenses administratives.

\section{Fonctionnement}

Au début d'un traitement, l'assuré doit consulter un médecin de premier recours conventionné sauf dans les cas suivants:

a) urgence, lorsque le médecin de premier recours n'est pas atteignable;

b) examens de la vue dans un but diagnostique ou thérapeutique;

c) contrôles gynécologiques et de grossesse;

d) consultation d'un spécialiste pour un problème spécifique, connu du médecin de premier recours (seconde opinion). Le spécialiste est tenu d'avertir rapidement le médecin de premier recours et convient avec lui des modalités de la prise en charge ultérieure.

Lorsqu'il adresse son patient à un spécialiste ou qu'il l'hospitalise, le médecin de premier recours envoie, à l'assurance, un avis de délégation pour 
l'informer de sa démarche, l'assureur n'honorant, sauf exception d) ci-dessus, que les honoraires de spécialiste pour lequel elle est en possession de ce document.

En cas de litige ou d'incompatibilité, le médecin de premier recours peut renoncer à la poursuite de la prise en charge de son patient, tout comme le patient vis-à-vis de son médecin. Dans les deux cas, les documents médicaux sont transmis au nouveau médecin.

Afin de respecter autant que possible le libre choix du médecin par une offre aussi large que possible, tous les internistes et généralistes exerçant à titre indépendant ont été invités à adhérer au Réseau.

Pour favoriser la qualité relationnelle de la prise en charge du patient, les actes médicaux sont rémunérés selon le tarif cadre, majoré de $10 \%$, les actes techniques selon le tarif cadre réduit de $10 \%$, le laboratoire conformément au tarif OFAS, ce qui représente également une diminution de $10 \%$. Globalement, ces divers ajustements respectent la neutralité des coûts, selon une simulation faite par la Caisse de médecins. Contrairement au système en vigueur à Genève, les médecins ont majoritairement décidé d'être rémunérés selon le principe du tiers-payant.

En adhérant au réseau, la prime mensuelle de l'assuré est réduite de $8 \%$, soit Fr. 25.-.

Les organes de contrôle sont constitués:

a) du Conseil médical, formé de trois membres des comités des médecins internistes et généralistes. Il décide de l'admission des médecins de premier recours après consultation du Conseil paritaire, supervise l'activité des médecins du Réseau et organise les cercles de qualité et sert d'organe arbitral en cas de litige entre médecins;

b) du Conseil des assureurs, constitué de trois représentants des caisses-maladie. Il décide de l'admission des caisses-maladie après consultation avec le Conseil paritaire, règle tous les problèmes de litiges entre les caissesmaladie du réseau;

c) du Conseil paritaire formé de la réunion du Conseil médical et du Conseil des caisses. Il élit son président en choisissant une personnalité neutre, n'ayant aucun lien entre les deux parties, en l'occurrence Madame Françoise Saudan, Conseillère aux Etats. Il surveille le fonctionnement économique du Réseau. Chaque caisse lui remet, à la fin de chaque exercice comptable, les données communiquées à l'OFAS. Il sert d'organe arbitral en cas de litige entre médecins et caisses-maladie et peut exclure un médecin ou une caisse du Réseau.
Les cercles de qualité, organisés par le Conseil médical, assurent, aux membres du Réseau, une formation continue par un travail d'auto-évaluation. Ils proposent des lignes de conduite applicables à des situations médicales courantes bien définies. Ils garantissent la valeur scientifique des schémas proposés et s'assurent qu'ils reposent sur des critères d'économicité. Ces lignes de conduite doivent être expliquées au patient afin qu'il puisse mieux adhérer aux démarches de son médecin traitant.

La participation aux cercles de qualité est reconnue comme formation continue obligatoire décidée par la FMH et les sociétés de discipline.

L'Association des Médecins du Canton de Genève (AMG) a donné son accord à la signature de cette Convention. Le code de déontologie de la FMH règle les rapports entre les médecins du réseau et les spécialistes.

Les médecins membres du Réseau s'acquittent d'une cotisation annuelle de Fr. 200.- devant couvrir les frais administratifs.

\section{Avatars}

La Convention définit, entre autres, le médecin de premier recours comme étant un généraliste, un interniste ou un pédiatre exerçant à titre indépendant. Cette précision avait pour but d'exclure les médecins travaillant dans des permanences, structures dans lesquelles les médecins pratiquent sous les ordres d'un médecin répondant lui-même dépendant d'un administrateur et ayant en général leur propre «réseau» de spécialistes. Un certain nombre de médecins, dont l'admission au Réseau a été refusée par le Conseil médical, a choisi de l'attaquer par voie juridique. Alors que la notion de médecin indépendant semble claire pour chacun d'entre nous, elle n'a aucune définition juridique. Le juge a donc cassé la décision du Réseau.

De plus, ce magistrat a relevé un vice juridique concernant notre Réseau, à savoir que, selon l'alinéa 4 de l'article 4 de la LAMal, seuls les assureurs (et pas les médecins) peuvent proposer aux assurés un choix limité de fournisseurs de prestations (par exemple sous forme de réseau). Ceci a eu pour conséquences:

1. la modification de la Convention par l'adjonction d'un Avenant (septembre 1997) stipulant que l'acceptation de l'adhésion d'un médecin au Réseau est du ressort des assureurs. Les médecins ont ainsi perdu le contrôle du Réseau. Ceux-ci doivent remplir des conditions précises pour y être admis, par exemple avoir un indice des coûts établi par le CAMS inférieur à 130; 
Tableau 1

Nombre d'internistes et généralistes ayant adhéré au Réseau.

\begin{tabular}{lccc} 
& 1998 & 1999 & 2000 \\
\hline Internistes & 213 & 208 & 206 \\
\hline Généralistes & 132 & 132 & 129 \\
\hline Total & 345 & 340 & 335
\end{tabular}

2. la mutation (décembre 1997) du RMIGG en ReMed (Association des médecins travaillant en Réseau).

\section{Résultats}

Le tableau 1 montre le nombre d'internistes, avec ou sans sous-spécialité et de généralistes avec ou sans FMH, ayant adhéré au Réseau durant les années 1998-2000. Ces médecins de premier recours représentent environ $80 \%$ de l'ensemble des généralistes genevois et $60 \%$ des internistes, cette différence de taux s'expliquant par le fait que de nombreux sous-spécialistes (gastroentérologues, oncologues, cardiologues, etc.) ne pratiquent plus la médecine interne et ne sont donc pas en mesure d'assumer la prise en charge globale d'un patient.

Le tableau 2 résume le nombre d'assurés Réseau et hors-Réseau des caisses-maladie partenaires à savoir Cosama (regroupant Assura, la Caisse vaudoise, le groupe Mutuel, Philos et Supra) et Intras pour cette même période, avec le pourcentage des assurés-Réseau par rapport aux assurés hors-Réseau. Ces caisses assurent 56\% de la population genevoise soumise à la LAMal. Considérée comme représentative des trois années, la figure 1 représente, à gauche, pour 1998, la répartition des assurés hors-Réseau pour les mineurs, les adultes femmes et hommes, et à droite, cette même répartition pour les assurés-Réseau. La différence du nombre de mineurs entre les assurés hors-Réseau (19\%) et Réseau (8\%), s'explique par le fait que les pédiatres n'ont pas adhéré au Réseau et que seuls quelques généralistes prennent en charge des enfants à partir de sept ans. Pour cette même raison, la moyenne d'âge des assurés-Réseau a été significativement plus élevées au cours des années 1998-1999 par rapport aux assurés hors-Réseau, cette donnée n'ayant pas été recensée pour 2000 (tab. 3). Ce même tableau souligne le fait que la proportion des assurés ayant opté pour la franchise ordinaire par rapport à la franchise à option est régulièrement plus élevée dans le groupe Réseau par rapport à celui hors-Réseau.

La figure 2 retrace le nombre d'assurés-Réseau par médecin.

Le tableau 4 stratifie, pour les deux groupes d'assurés, la moyenne par assuré, pour les mineurs, les femmes et les hommes, du montant des prestations brutes, des participations nettes et enfin, de la moyenne des prestations nettes, correspondant au montant des prestations versées par les assureurs aux divers prestataires de soins ou de services.

Le tableau 5 compare, pour les deux groupes d'assurés et par sexe, les prestations de pharmacie et de laboratoire pour l'année 2000, ces données n'étant pas disponibles pour les années 1998 et 1999.

Les assureurs nous ont fourni les comptes des produits et charges des assurés-Réseau mais pas ceux des assurés hors-Réseau.

\section{Discussion}

La Suisse vit chaque automne, lors de l'annonce de l'augmentation des primes d'assurance maladie, le spectacle affligeant des divers acteurs de la

Tableau 2

Assurés-Réseau et hors-Réseau des Caisses partenaires.

\begin{tabular}{|c|c|c|c|c|c|c|c|c|c|}
\hline & \multirow{2}{*}{\multicolumn{2}{|c|}{ 1.1.1998 }} & \multirow[b]{3}{*}{ \% Réseau } & \multicolumn{3}{|l|}{ Assurés } & \multirow{2}{*}{\multicolumn{2}{|c|}{1.1 .2000}} & \multirow[b]{3}{*}{ \% Réseau } \\
\hline & & & & 1.1.1999 & & & & & \\
\hline & Réseau & Hors-Réseau & & Réseau & Hors-Réseau & \% Réseau & Réseau & Hors-Réseau & \\
\hline Assura & 874 & 33809 & 2,52 & 1030 & 34414 & 2,91 & 1137 & 38526 & 2,87 \\
\hline La Caisse VD & 1704 & 15409 & 9,96 & 1681 & 15237 & 9,94 & 1786 & 15549 & 10,30 \\
\hline Groupe Mutuel & 1126 & 50281 & 2,19 & 2023 & 60352 & 3,24 & 3225 & 64659 & 4,75 \\
\hline Philos & 617 & 1086 & 36,23 & 710 & 1018 & 41,09 & 729 & 1077 & 40,37 \\
\hline Supra & 1835 & 20216 & 8,32 & 2047 & 18822 & 9,81 & 1657 & 16620 & 9,07 \\
\hline Intras & 1831 & 43933 & 4,00 & 1626 & 55000 & 2,87 & 1790 & 45687 & 3,77 \\
\hline Total & 7987 & 164734 & 4,62 & 9117 & 184843 & 4,70 & 10324 & 182118 & 5,36 \\
\hline
\end{tabular}


Figure 1 gauche

Répartition des assurés-Réseau en 1998.

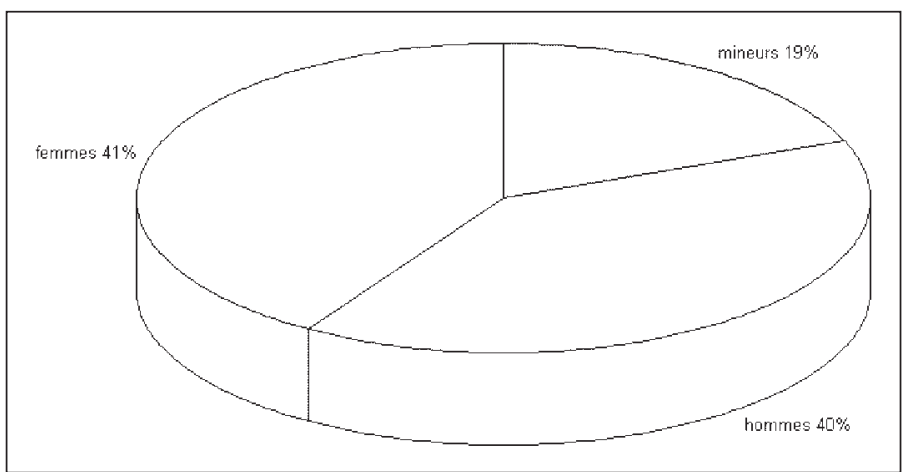

Figure 1 droite

Répartition des assurés non-Réseau en 1998.

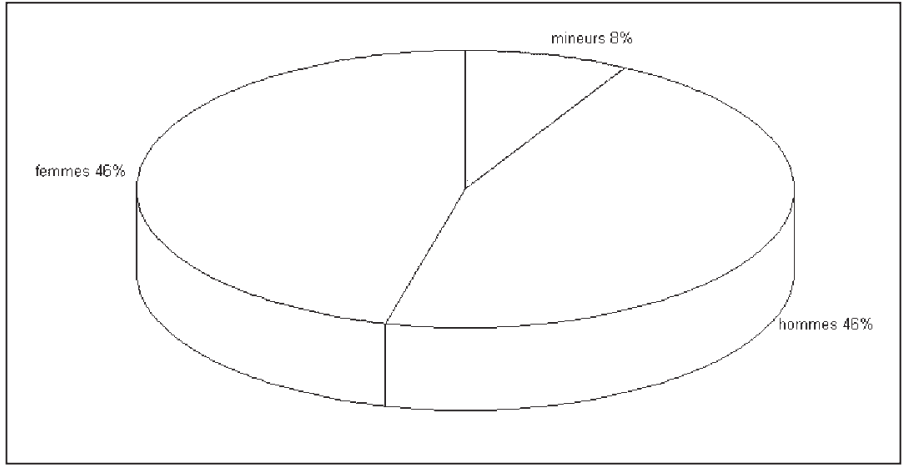

santé s'en rejetant mutuellement la responsabilité. A cela s'ajoute la litanie des autorités fédérales promettant que la LAMal finira par apporter ses effets bénéfiques à la maîtrise des coûts.

Le groupe des internistes et généralistes genevois a cherché, en développant un «banc d'essai», le Réseau, à savoir si quelques mesures simples et concrètes auraient un effet modérateur sur les coûts de la santé. Quelles sont ces mesures?

1. revenir à la notion de médecin de famille, pivot de notre système de santé, qui ne doit pas être un «gate keeper» mais au contraire un praticien capable d'assumer une prise en charge globale du patient;

2. sensibiliser le médecin aux coûts de la santé en insistant sur l'importance de l'écoute, de l'examen clinique et de l'utilisation rationnelle des moyens techniques;

3. introduire la notion de cercles de qualité visant à mettre sur pied des lignes de conduite et favoriser l'échange d'informations,

4. renforcer la relation de confiance du patient en son médecin-traitant, évitant qu'il consulte plusieurs thérapeutes pour la même affection;

5. établir un dialogue constructif avec les assureurs pour évaluer les avantages et les inconvénients d'un tel concept.

Soulignons d'emblée que ce Réseau s'est voulu très libéral en acceptant, à de rares exceptions près (médecins non indépendants ou n'ayant pas une formation suffisante), tous les médecins internistes et généralistes, ainsi que tous les assureurs intéressés à cette forme d'assurance. Le très grand nombre de médecins garantissait au patient de pouvoir continuer à être suivi par son médecin habituel. L'accès au spécialiste était possible, pour un avis, en cas de divergence avec le médecin-traitant.

L'intérêt d'une telle formule, la seule actuellement innovante, est très variable d'une caisse à l'autre: le tableau 2 montre que Philos, caisse assurant une bonne promotion du Réseau auprès d'une population assez homogène de professionnels de la terre, parvient à convaincre 40\% de ses adhérents alors qu'à l'opposé, telle autre qui ne le soutient que «mollement» recrute moins de $3 \%$ de ses assurés.

Le même parallèle peut se faire pour les médecins (fig. 2). Ceux-ci ont reçu un matériel d'information (affichettes, formulaires d'inscription) à disposition de leurs patients dans leur salle d'attente. Beaucoup ont fait la promotion du Réseau auprès de leurs patients (70 médecins soignent plus de 20 patients-Réseau) alors que d'autres estimaient que tel n'était par leur rôle (100 en traitent moins que 5).

La population d'assurés-Réseau a relativement peu augmenté durant ces trois années, passant de 4,62\% à 5,36\% de l'ensemble des assurés. Deux explications peuvent être avancées: d'une part, la promotion du Réseau a été essentiellement faite par les médecins, d'autre part, l'incitatif financier (Fr. 25.- par mois) est souvent jugé insuffisant par les assurés.

Tableau 3

Moyenne d'âge des assurés-Réseau et hors-Réseau. Pourcentage des franchises ordinaires par rapport aux franchises à option.

\begin{tabular}{|c|c|c|c|c|c|c|}
\hline Assurés & $\begin{array}{l}1998 \\
\text { Réseau }\end{array}$ & Hors-Réseau & $\begin{array}{l}1999 \\
\text { Réseau }\end{array}$ & Hors-Réseau & $\begin{array}{l}2000 \\
\text { Réseau }\end{array}$ & Hors-Réseau \\
\hline Age moyen (années) & 41 & 36 & 41 & 35 & non & disponible \\
\hline Franchise ordinaire / franchise à option (\%) & 18 & 28 & 20 & 30 & 22 & 31 \\
\hline
\end{tabular}


Tableau 4

Montant des prestations brutes, des participations et des prestations nettes pour les assurés-Réseau et hors-Réseau.

\begin{tabular}{|c|c|c|c|c|c|c|c|}
\hline & & 1998 & & 1999 & & 2000 & \\
\hline & & Réseau & Hors-Réseau & Réseau & Hors-Réseau & Réseau & Hors-Réseau \\
\hline \multirow[t]{2}{*}{ Hommes } & prestations & 1475 & 1981 & 1789 & 2101 & 2004 & 2447 \\
\hline & participations & 332 & 349 & 399 & 373 & 413 & 409 \\
\hline \multirow[t]{2}{*}{ Femmes } & prestations & 2431 & 2870 & 2744 & 3025 & 3104 & 3697 \\
\hline & participations & 475 & 486 & 536 & 497 & 571 & 555 \\
\hline \multirow[t]{2}{*}{ Mineurs } & prestations & 975 & 1074 & 918 & 962 & 800 & 1008 \\
\hline & participations & 184 & 155 & 151 & 141 & 142 & 136 \\
\hline Prestations nettes & & 1494 & 1805 & 1734 & 1859 & 1969 & 2240 \\
\hline
\end{tabular}

Les résultats financiers ne sont pas faciles à évaluer, tant les paramètres intervenant dans leur établissement sont complexes. Comme relevé ci-dessus, l'adhésion de patients s'est faite pour une grande part sur le conseil de leur médecin. Il s'agit, a priori d'une population «malade» et plus âgée (âge moyen des assurésRéseau 41 ans versus 36 ans pour les assurés horsRéseau). Cette affirmation est corroborée par une dépense plus élevée pour les médicaments dans le collectif Réseau (respectivement 594.- pour les femmes et 466.- pour les hommes) en comparaison aux assurés hors-Réseau (562.- pour les femmes et 443.- pour les hommes), alors que les génériques sont censés être plus souvent prescrits aux patients-Réseau. La limitation aux patients-Réseau des examens de laboratoire s'est vérifiée (respectivement 167.- pour les femmes

Figure 2

Classement par médecin du nombre de patients-Réseau.

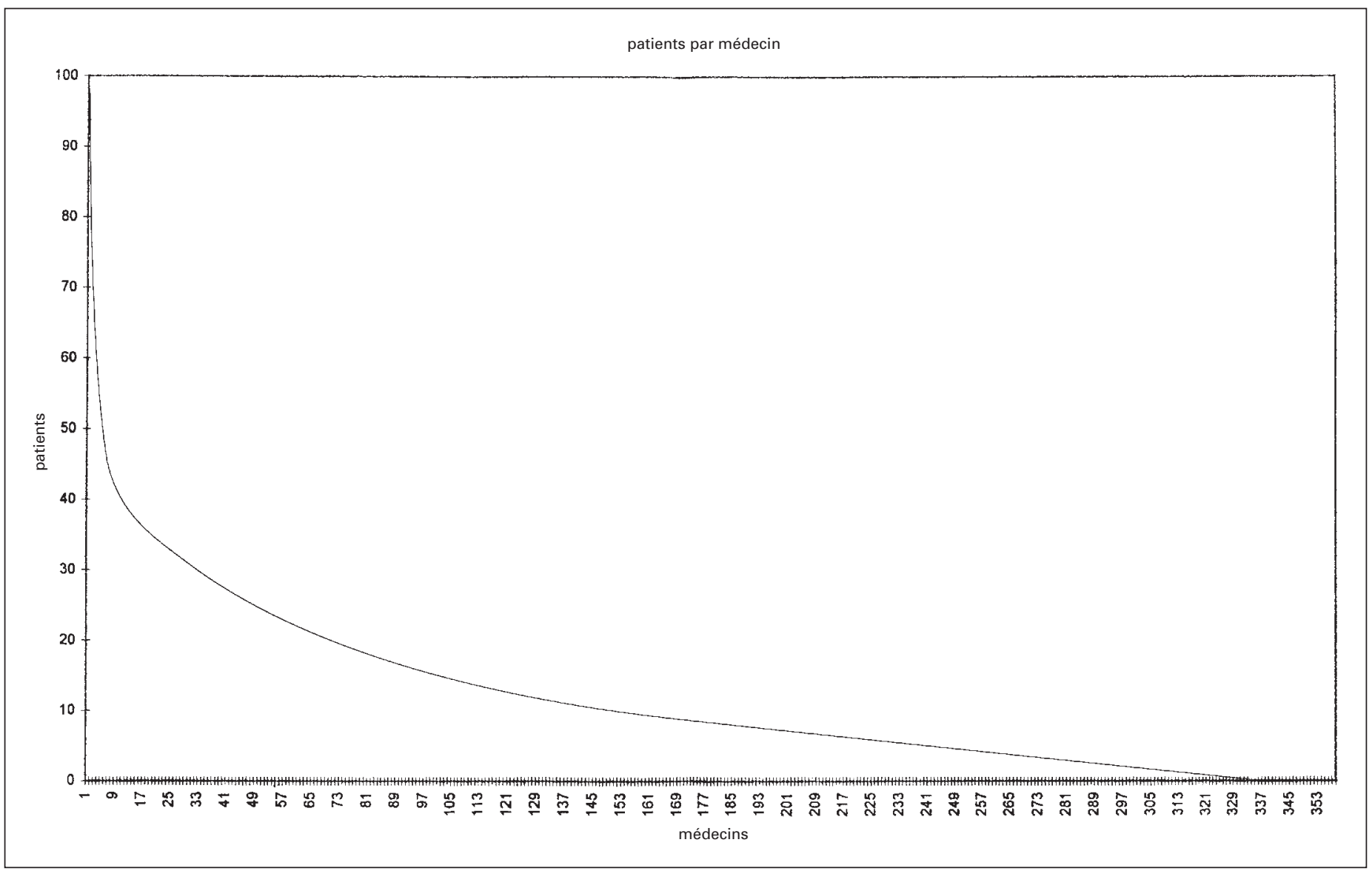


Tableau 5

Prestations par assurés femmes et hommes pour la pharmacie et le laboratoire en 2000 .

\begin{tabular}{lllll} 
& $\begin{array}{l}\text { femmes } \\
\text { Réseau }\end{array}$ & Hors-Réseau & $\begin{array}{l}\text { hommes } \\
\text { Réseau }\end{array}$ & Hors-Réseau \\
\hline Pharmacie (Fr.) & 594 & 562 & 466 & 443 \\
\hline Laboratoire (Fr.) & 167 & 177 & 70 & 86
\end{tabular}

et 70.- pour les hommes, versus 177.- pour les femmes et 86.- pour les hommes hors-Réseau).

Enfin, il faut relever que, les assurés-Réseau étant en tiers payant, toutes les notes d'honoraires les concernant, mêmes celles inférieures à la franchise, ont été envoyées par les prestataires de soins à la caisse maladie, ce qui n'était pas le cas des assurés hors-Réseau couverts en tiers garant, par ailleurs au bénéfice d'une franchise plus élevée. En conséquence, un certain nombre de factures dont le montant était inférieure à la franchise n'a jamais été envoyé à la caisse maladie contribuant ainsi à réduire la dépense des assurés hors-Réseau.

$\mathrm{Au}$ cours de ces trois années, le différentiel des prestations nettes en faveur des assurésRéseau s'est établi à de Fr. 310.- par assuré en 1998, à 125.- en 1999 et à 271.- en 2000, soit une réduction annuelle des coûts respectivement de $17 \%, 7 \%$ et $12 \%$.

En marge de ces considérations financières, il convient de souligner le très grand succès rencontré par la mise sur pied des cercles de qualités, lieu privilégié d'échanges et de formation. Enfin, quoique réticents au début, les spécialistes ont compris que le Réseau ne cherchait pas à les «court-circuiter» mais au contraire à leur envoyer des patients pour lesquels leur intervention était réellement nécessaire.

\section{Conclusions}

Notre Réseau de médecin de premiers recours, qui se différencie totalement d'une HMO, a atteint son objectif à savoir de permettre une réduction substantielle des coûts de la santé tout en préservant une quasi liberté de choix du médecin-traitant et en assurant des soins de qualité. Ce système ne peut se développer qu'en partenariat entre assurés, assureurs, soignants et pouvoir politique. Ce dernier acteur n'a pas été directement inclus dans notre expérience pour éviter toute critique idéologique. Il est certain qu'il ne pourra être tenu à l'écart dans l'avenir. Le Réseau vise à renforcer la relation de confiance entre patient et médecin-traitant. Il ne cherche, en aucun cas, à éliminer le spécialiste de la prise en charge du patient. En rationalisant les soins, il évite le développement d'une médecine «à deux vitesses».

\section{Remerciements}

Je tiens à remercier chaleureusement tous ceux qui ont rendu possible cette expérience:

- Mme Françoise Saudan, Présidente du Conseil paritaire du Réseau;

- le Dr Claude Aubert, ex-Président de l'Association des médecins du canton de Genève;

- les assureurs de la Cosama et d'Intras;

- les membres de mon comité: les Drs J. C. Canavese, F. Frésard, F. Maggi, F. Stroot, A. Vilaseca, C. Witz et T. Wyss. 